\title{
A two-dimensional MHD model of the solar wind interaction with Mars
}

\author{
H. Shinagawa ${ }^{1}$ and S. W. Bougher ${ }^{2}$ \\ ${ }^{1}$ Solar-Terrestrial Environment Laboratory, Nagoya University, Toyokawa 442-8507, Japan \\ ${ }^{2}$ Lunar and Planetary Laboratory, University of Arizona, Tucson, Arizona 85721, U.S.A.
}

(Received August 7, 1997; Revised June 20, 1998; Accepted August 18, 1998)

\begin{abstract}
The ionosphere of Mars is expected to be significantly affected by the solar wind because Mars does not possess a significant intrinsic magnetic field which deflects the solar wind. Despite a number of plasma measurements made near Mars, the nature of the solar wind-Mars interaction has not yet been fully understood. In order to self-consistently study the solar wind interaction with the ionosphere of Mars, a two-dimensional MHD model has been developed with an emphasis placed on the structure of the ionosphere of Mars. It is found that the modeled electron density profile in the upper ionosphere strongly depends on the solar wind dynamic pressure as well as the solar zenith angle. The ionosphere in the model tends to have an ionopause-like sharp drop of the electron density at some altitude for realistic solar wind dynamic pressures. Such behavior is not consistent with most of the observed electron density profiles, which exhibit relatively large and constant scale height in most of the dayside region. While the observed electron density profiles of the Venus ionosphere have been reproduced reasonably well by ionospheric models as well as recent three-dimensional MHD models, the electron density profiles of the Martian ionosphere have not been successfully reproduced by theoretical models including this study. This fact implies that processes not present in the Venus ionosphere, such as crustal magnetic fields and the rotation of the planet, may have significant effects on the structure and the dynamics of the ionosphere of Mars.
\end{abstract}

\section{Introduction}

A number of electron density profiles of the Martian ionosphere have been obtained by radio occultation measurements made by the Mariner and Viking orbiters (cf. Zhang et al., 1990; Kliore, 1992). Before the Mars Global Surveyor arrived at Mars, in situ measurements had been made only by the Viking landers, and the magnetic field had not been measured in the ionosphere. Recently the Mars Global Surveyor has detected a fairly strong ( $B \leq 400 \mathrm{nT}$ ) but localized magnetic field which is thought to be of crustal origin (Acuña et al., 1998). It has also been found that the surface magnetic field of core origin is likely to be smaller than about $5 \mathrm{nT}$, indicating that a global intrinsic magnetic field at Mars is unlikely to play an important role in the solar wind interaction with Mars.

A one-dimensional (1D) ionospheric model of Mars which includes the ionospheric magnetic field was developed by Shinagawa and Cravens $(1989,1992)$. In their model, the neutral density model based on the Viking 1 measurement was used. The observed ion density profiles were reproduced fairly well by the model, and the results suggested that an intrinsic magnetic field at Mars is insignificant. However, the 1D ionospheric model is not capable of self-consistently including horizontal transport processes which are driven by the horizontal pressure gradient force as well as by the electromagnetic force generated by the solar wind. The other problem is that the model has an upper boundary at a given altitude $(480 \mathrm{~km})$, and therefore the upper boundary condi-

Copy right(C) The Society of Geomagnetism and Earth, Planetary and Space Sciences (SGEPSS); The Seismological Society of Japan; The Volcanological Society of Japan; The Geodetic Society of Japan; The Japanese Society for Planetary Sciences. tions must have been given from the observations and theoretical consideration. The upper boundary condition was also a problem with a two-dimensional (2D) ionospheric MHD model of Shinagawa (1996).

In order to model the structure of the Martian ionosphere including the solar wind interaction, we developed a 2D MHD model of the solar wind-Mars interaction. The model includes the ionosphere of Mars in more realistically than previous global models of solar wind-Mars interaction. The upper boundary has been extended to about seven radii from the center of Mars. There have been a number of studies of the solar wind-Venus interaction, suggesting that the ionospheric structure of the non-magnetized planets is controlled mainly by the solar wind dynamic pressure $\left(P_{\mathrm{sw}}\right)$. Thus, two basic cases are examined in this paper: (1) high $P_{\mathrm{sw}}$, and (2) low $P_{\text {sw }}$.

It must be noted that $2 \mathrm{D}$ modeling is not enough to accurately incorporate effects associated with three-dimensional (3D) configuration, such as tension of the magnetic field, horizontal transport of plasma along magnetic field lines. To study the interaction between the solar wind and the nonmagnetized planets including such effects, 3D modeling is necessary. Recently, several groups developed 3D MHD models of the solar wind-Venus interaction (McGary and Pontius, 1994; Cable and Steinolfson, 1995; Murawski and Steinolfson, 1996a,b; Tanaka and Murawski, 1997). Among these studies, Tanaka and Murawski (1997) included the ionosphere of Venus and the solar wind simultaneously. The bow shock, the magnetic barrier, and the ionopause were reproduced realistically. The dynamics of the ionosphere of Venus was also reproduced reasonably well. Most recently, Tanaka (1998) has studied the solar wind interaction with 
the ionospheres of non-magnetized planets for two different solar wind conditions using a 3D MHD model with a two-component plasma. Tanaka and Murawski (1997) and Tanaka (1998) reported that the tension force becomes important near the terminator region. However, present 3D models treat the ionosphere in a very much simplified manner, and have not included the lower ionosphere, or $\mathrm{O}_{2}^{+}$ionosphere. We believe that 2D modeling still gives useful ideas in studying the ionosphere of Mars and its interaction with the solar wind.

\section{Model Description}

The 2D time-dependent single-fluid MHD equations, i.e., the continuity, momentum, energy, and magnetic induction equations are solved in a cylindrical coordinate system as shown in Fig. 1. The upper boundary is set at $25000 \mathrm{~km}$ from the center of Mars. The lower boundary is at $100 \mathrm{~km}$ above the surface, which is nearly the bottom of the ionosphere. Calculations are done for the upper half of the hemisphere, assuming axial symmetry with respect to the sun-Mars line. The left boundary is at the subsolar point, i.e., $0^{\circ}$ solar zenith angle (SZA), and the right boundary is at the antisolar point, i.e., $\mathrm{SZA}=180^{\circ}$. The lower boundary conditions are given at $100 \mathrm{~km}$, because at this altitude (i) photochemical equilibrium is a reasonable assumption for density, (ii) the ion velocity is considered to be the same as the neutral velocity which is assumed to be zero in this study, (iii) the plasma temperature is the same as the neutral temperature because of very large ion-neutral and electron-neutral collision frequencies, and (iv) the magnetic field at $100 \mathrm{~km}$ is zero. In the upper boundary condition, the density, velocity, magnetic field, and temperature of the solar wind are given in the upstream side, and gradients are taken to be zero for all variables in the downstream side.

The basic method of the modeling is the same as that of Shinagawa (1996). The solar wind magnetic field is assumed

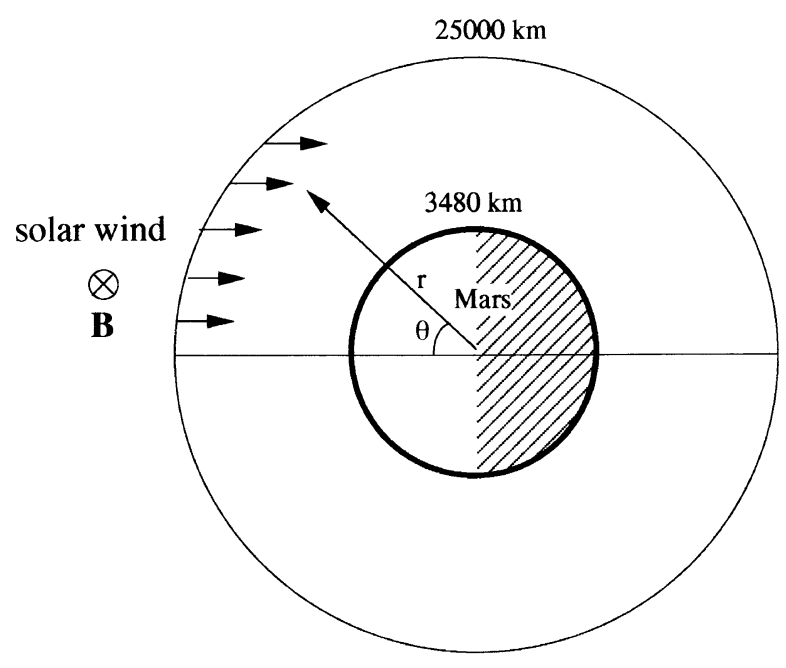

Fig. 1. Coordinate system and region of the model. The cylindrical coordinate is used. The direction of the solar wind magnetic field is in $z$ axis and into the paper. The upper boundary is at $25000 \mathrm{~km}$, and the lower boundary is at $3480 \mathrm{~km}$ which is $100 \mathrm{~km}$ above the surface of Mars. to be perpendicular to the plane. In the continuity equation, the mass continuity equation is also included in addition to the number density equation. It is assumed that ions produced in the ionosphere have $16 \mathrm{amu}$, i.e., mass of $\mathrm{O}^{+}$. The electron density is expressed as the sum of the $\mathrm{O}^{+}$density and the $\mathrm{O}_{2}^{+}$density. This procedure enables us to approximately separate solar wind ions $\left(\mathrm{H}^{+}\right)$and ionospheric ions $\left(\mathrm{O}^{+}\right)$. The ionosphere observed by the Viking landers indicated that the ions in the ionosphere consist of both $\mathrm{O}_{2}^{+}$and $\mathrm{O}^{+}$(Hanson et al., 1977). If $\mathrm{O}_{2}^{+}$is included in the model, the scale height of the electron density would be slightly smaller. Artificial viscosity terms are added to all equations to maintain numerical stability.

Ionospheric parameters, such as ionization rates, chemical reaction rates, are basically the same as those used for 1D model (Shinagawa and Cravens, 1989, 1992), i.e., the parameters under the Viking conditions. A weak artificial ionization source (ionization rates are $10^{-4}$ of the dayside values) is given on the nightside to prevent numerical instability caused by extremely small densities. The radial grid size varies from $4 \mathrm{~km}$ near the lower boundary up to $500 \mathrm{~km}$ near the upper boundary. The angular grid size is $2.5^{\circ}$ for all SZAs. The grid size is $4 \mathrm{~km}$ at altitudes less than $480 \mathrm{~km}$ in vertical direction, which is enough to reproduce a fairly thin ionopause with a thickness of about $20 \mathrm{~km}$. Effects of the rotation of Mars are not included in this study.

Two cases, Case 1 (high $P_{\mathrm{sw}}$ ) and Case 2 (low $P_{\mathrm{sw}}$ ), are examined. For both Case 1 and Case 2, the solar wind plasma density, temperature, and magnetic field are taken to be $5 \mathrm{~cm}^{-3}, 2 \times 10^{5} \mathrm{~K}$, and $3 \mathrm{nT}$, respectively. The solar wind velocity $V_{\mathrm{sw}}$ is taken to be $450 \mathrm{~km} / \mathrm{s}$ for Case 1 and $300 \mathrm{~km} / \mathrm{s}$ for Case 2. Starting from practically zero densities in the modeling region, the system approximately reached a steady state in about three hours.

\section{Results}

\subsection{Case 1 (High $\boldsymbol{P}_{\text {sw }}$ )}

The solar wind velocity $\left(V_{\mathrm{sw}}\right)$ is $450 \mathrm{~km} / \mathrm{s}$, and the solar wind density is $5 \mathrm{~cm}^{-3}$, giving a solar wind dynamic pressure $\left(P_{\mathrm{sw}}\right)$ of about $1.7 \times 10^{-8}$ dyne $/ \mathrm{cm}^{2}$. Under this condition, $P_{\mathrm{sw}}$ considerably exceeds the maximum ionospheric thermal pressure $\left(P_{\mathrm{i}}\right)$ of Mars which is about $5 \times 10^{-9}$ dyne $/ \mathrm{cm}^{2}$ in this model. The Pioneer Venus observations confirmed that in the solar wind-Venus interaction for the case of $P_{\mathrm{sw}}>P_{\mathrm{i}}$, most of the dayside ionosphere becomes strongly magnetized and compressed, and the ionopause height becomes low (cf. Elphic et al., 1981). This type of the ionosphere has been reproduced by both 2D and 3D models reasonably well (Shinagawa, 1996; Tanaka and Murawski, 1997; Tanaka, 1998).

The modeled ionosphere of Mars appears to be analogous to the Venus ionosphere for $P_{\mathrm{sw}}>P_{\mathrm{i}}$. Figure 2 shows the contour plot of the calculated electron densities around Mars in logarithm of particle number in cubic centimeters. The solar wind is flowing from the left-hand side to the righthand side. A bow shock is formed at about $1.4 R_{\mathrm{M}}$, which is roughly the same as the observed values (Slavin and Holzer, 1982; Schwingenschuh et al., 1990), although the 2D model is not capable of accurately reproducing the position and shape of the bow shock. The ionosphere is very thin, and 


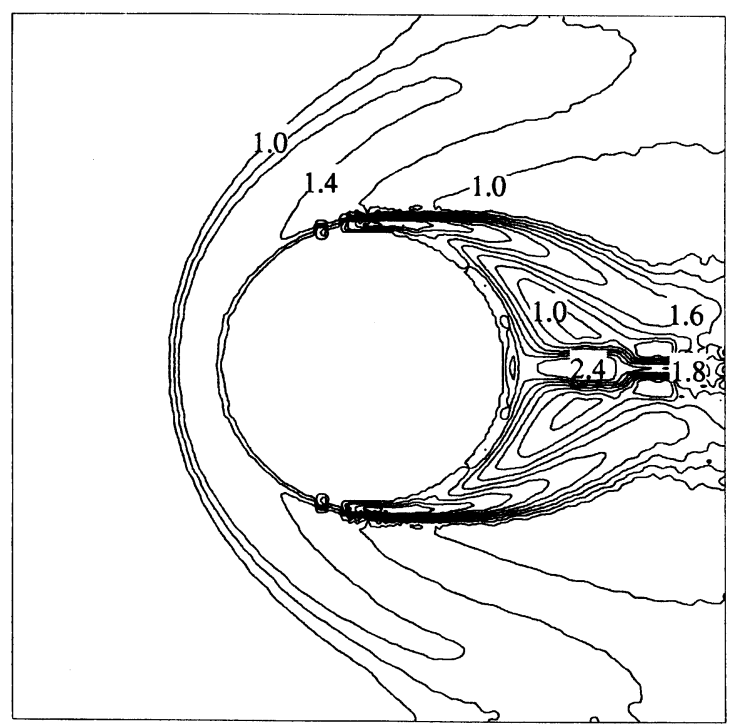

Fig. 2. Contour plot of the calculated electron densities in cubic centimeters in logarithmic scale for Case 1. Contour lines are not plotted near the surface of Mars $(z<500 \mathrm{~km})$.

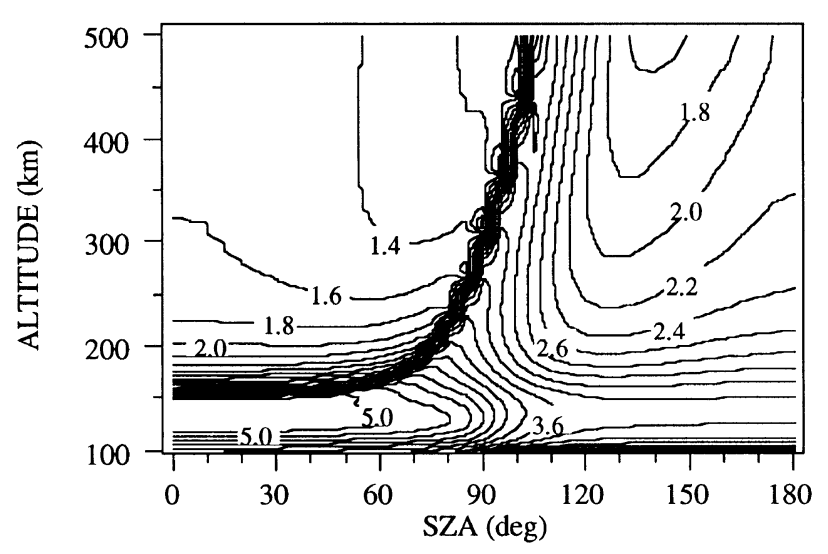

Fig. 3. Contour plot of the calculated electron densities below $500 \mathrm{~km}$ in cubic centimeters in logarithmic scale for Case 1.

cannot be seen in Fig. 2. On the nightside, a weak ionosphere with the electron density of $10^{1}-10^{2} \mathrm{~cm}^{-3}$ extends up to the wake region, forming an "ionotail". The overall plasma structure in this model is similar to that of the 3D study by Tanaka (1998).

The calculated electron density in the ionosphere is shown in the contour in Fig. 3. Ions in the topside ionosphere are removed by strong downward and day-to-night convection driven by the solar wind dynamic pressure. It is found that an ionopause is formed at an altitude of about $170 \mathrm{~km}$ from $\mathrm{SZA}=0^{\circ}$ to $\mathrm{SZA}=60^{\circ}$. The ionopause height increases rapidly with $\mathrm{SZA}$ for $\mathrm{SZA}>60^{\circ}$. The "low-altitude ionopause" is commonly observed in the Venus ionosphere when the solar wind dynamic pressure exceeds the ionospheric thermal pressure (Elphic et al., 1981). In the terminator region, there is a small day-to-night ion transport,

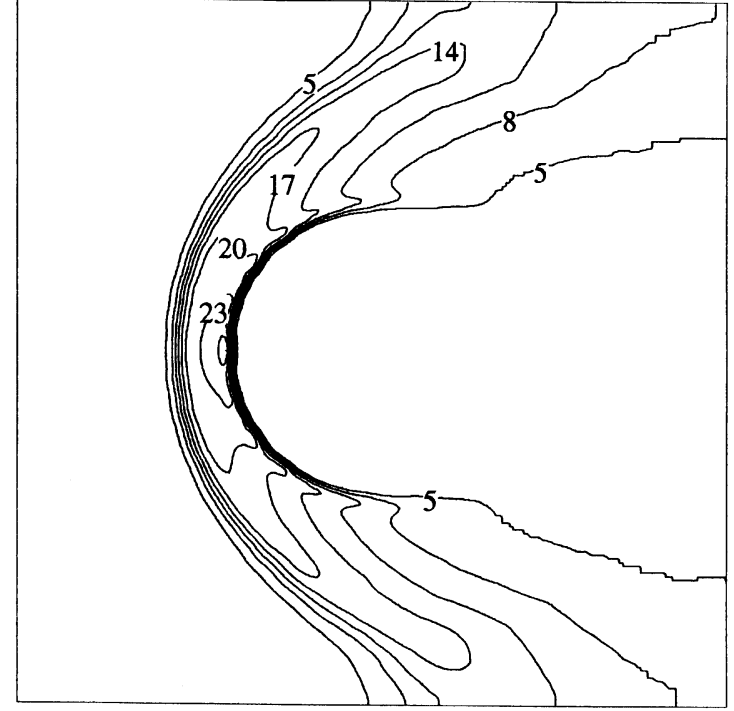

Fig. 4. Contour plot of the calculated magnetic field strength in nanotesla for Case 1 .

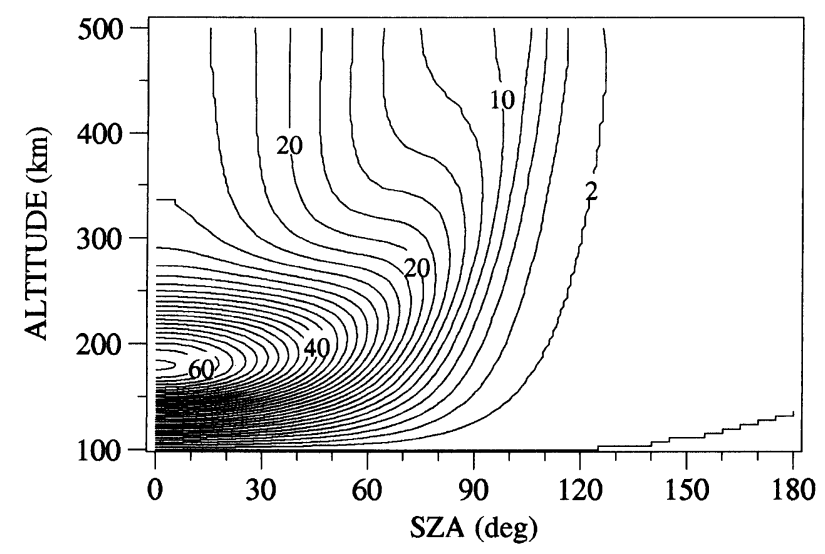

Fig. 5. Contour plot of the magnetic field strength in nanotesla at altitudes below $500 \mathrm{~km}$ for Case 1 .

but it is not enough to maintain the significant nightside ionosphere. A weak nightside ionosphere of $10^{3} \mathrm{~cm}^{-3}$ is maintained primarily by artificially ionization source in this model. This is not necessarily unrealistic because in the case of the nightside ionosphere of Venus the electron density of $10^{3}-10^{4} \mathrm{~cm}^{-3}$ is thought to be maintained by ionization due to particle precipitation.

The magnetic field distribution around Mars is shown in Fig. 4. In the post bow shock region, i.e., the magnetosheath, the magnetic field strength is enhanced, and reaches about $20 \mathrm{nT}$. The calculated magnetic field strength in the ionosphere is shown in contours in Fig. 5. The magnitude of the ionospheric magnetic field reaches the maximum of about $60 \mathrm{nT}$ at the altitude of $170 \mathrm{~km}$ in the subsolar region. This maximum is created by pileup of the magnetic flux from the higher region to the lower region where the vertical plasma flow slows down through ion-neutral drag force. At the bot- 


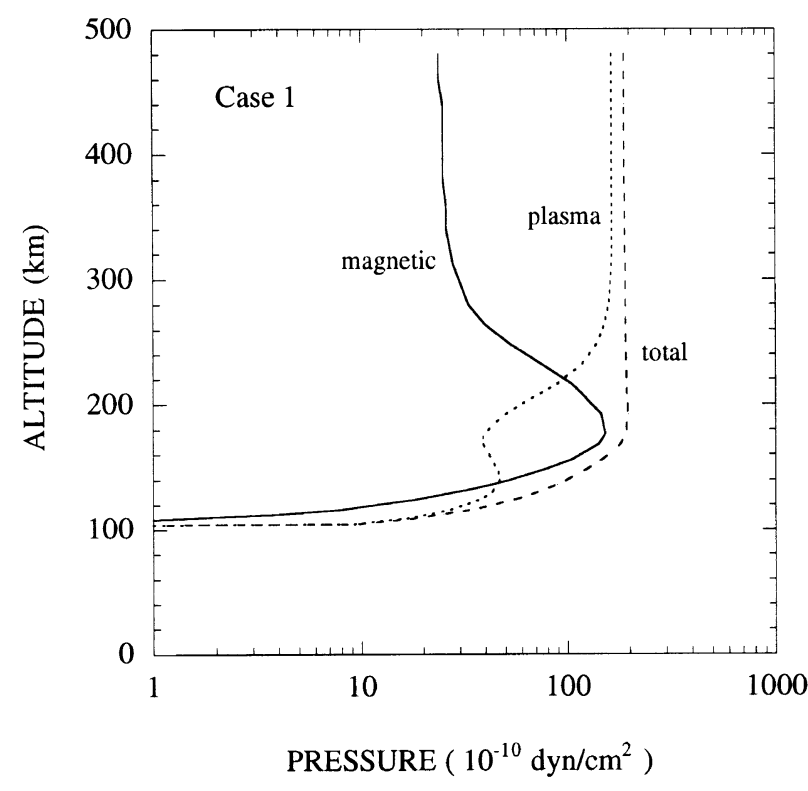

Fig. 6. Altitude profile of the plasma thermal pressure, the magnetic pressure, and the total pressure in the ionosphere for Case 1 in the unit of $10^{-10}$ dyne $/ \mathrm{cm}^{2}$.

tom of the ionosphere, the magnetic flux is removed by the magnetic diffusion because of large resistivity. The magnetic field strength decreases rapidly with SZA, and becomes nearly zero in the nightside ionosphere. A weak magnetic field in the region beyond the terminator is the result of a numerical diffusion effect on the magnetic field.

The altitude profiles of the plasma thermal pressure, the magnetic pressure, and the total pressure at the subsolar point are shown in Fig. 6. The pressure is in the form of plasma thermal pressure above $200 \mathrm{~km}$. In this region, the plasma density is low, but the temperature is high. Below $200 \mathrm{~km}$ temperature becomes low because of thermal energy is lost by the ion-neutral collisions. The magnetic pressure becomes high at about $170 \mathrm{~km}$. The magnetic field is removed through magnetic diffusion at low altitudes. The total pressure gradient force is balanced with the ion-neutral drag force at the bottom of the ionosphere. The solar wind dynamic pressure is converted to the plasma thermal pressure and the magnetic pressure at the bow shock. Then, the magnetic pressure becomes dominant in the lower magnetosheath region which is sometime called the magnetic barrier region. In the lower ionosphere, the magnetic pressure is balanced with the ionospheric thermal pressure plus the ion-neutral drag force.

\subsection{Case 2 (Low $P_{\text {sw }}$ )}

A case for the solar wind velocity of $300 \mathrm{~km}$ and the density of $5 \mathrm{~cm}^{-3}$, is also investigated. The dynamic pressure is now about $7.5 \times 10^{-9}$ dyne $/ \mathrm{cm}^{2}$, which is lower and more common than Case 1. The electron density in this ionosphere for this case is shown in the contour plot of Fig. 7. The height of ionopause is about $180 \mathrm{~km}$ in the subsolar region, and increases rapidly with SZA because of rapid decrease of ram $P_{\mathrm{sw}}$ exerting on the ionosphere. The ionopause appears at higher altitudes at all SZAs than that of Case 1, but the thickness is still relatively small. The ionospheric thermal

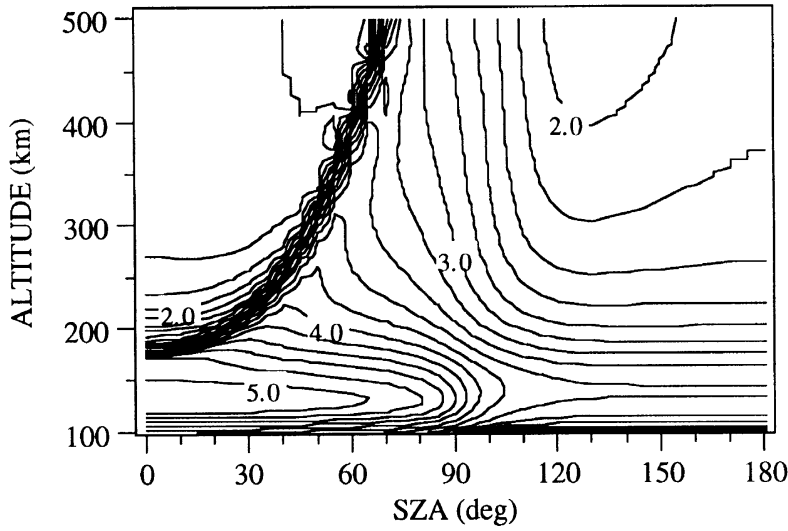

Fig. 7. Contour plot of the calculated electron densities below $500 \mathrm{~km}$ in cubic centimeters in logarithmic scale for Case 2.

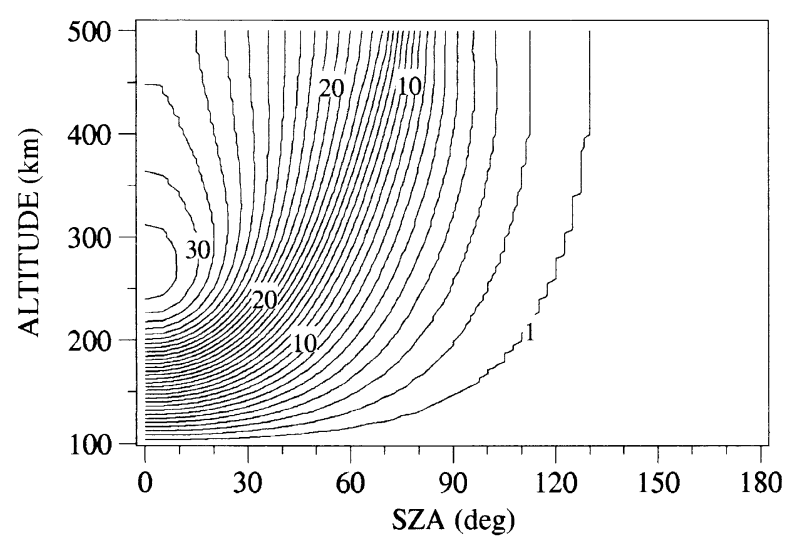

Fig. 8. Contour plot of the magnetic field strength in nanoteslas at altitudes below $500 \mathrm{~km}$ for Case 2 .

pressure is now sufficient to hold the solar wind dynamic pressure except in the subsolar region.

There is a day-to-night ion transport, but unlike the Venus ionosphere, the ion flux is smaller mainly because of the lower density in the dayside ionosphere. The ion density in the nightside ionosphere is of the order of $10^{3} \mathrm{~cm}^{-3}$. The calculated magnetic field in the ionosphere is shown in the contour plot (Fig. 8). The maximum of the magnetic field strength is now $30 \mathrm{nT}$ at about $270 \mathrm{~km}$. The overall behavior of the magnetic field for Case 2 is similar to that for Case 1 .

The altitude profile of the plasma thermal pressure, the magnetic pressure, and the total pressure is shown in Fig. 9. Compared with Case 1, the plasma thermal pressure at high altitudes is lower the magnetic pressure above $200 \mathrm{~km}$. The magnetic pressure is held by the ionospheric thermal pressure below $200 \mathrm{~km}$. Then, the total pressure gradient force is balanced with the ion-neutral drag force. The distribution of the pressure profiles is qualitatively consistent with that obtained by the 3D MHD model of the solar wind interaction with non-magnetized model (Tanaka, 1998). 


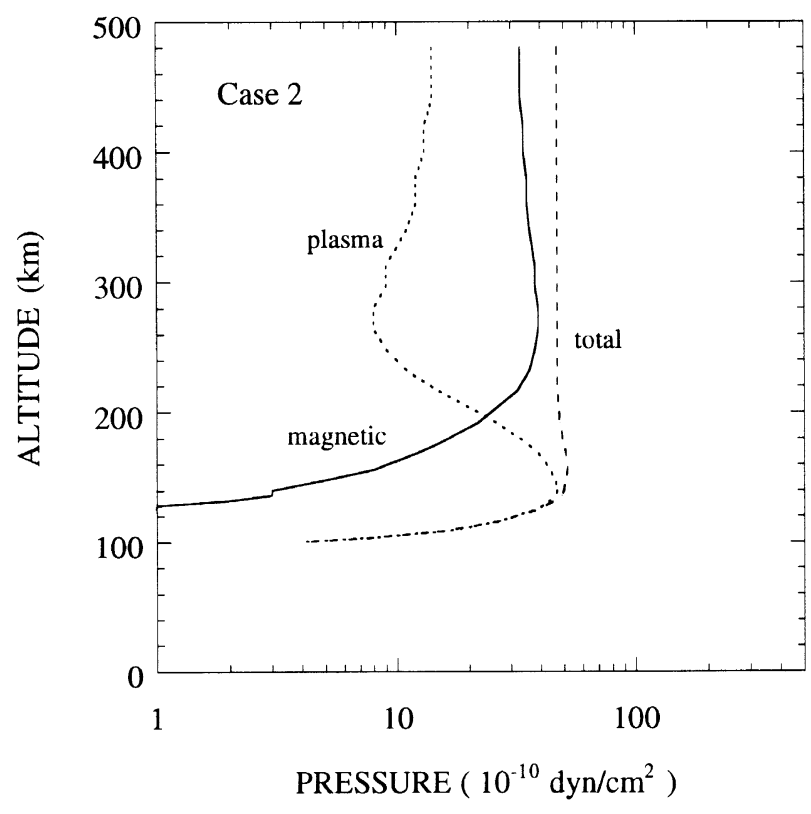

Fig. 9. Altitude profile of the plasma thermal pressure, the magnetic pressure, and the total pressure in the ionosphere for Case 2 in the unit of $10^{-10}$ dyne $/ \mathrm{cm}^{2}$

\section{Discussion}

The Viking observations confirmed that the ionospheric peak plasma pressure observed by the Viking landers is significantly smaller than the average solar wind dynamic pressure, indicating that the ionospheric magnetic field compensates for the missing pressure at least under the Viking conditions (Hanson and Mantas, 1988). The question then was whether this ionospheric magnetic field is an intrinsic magnetic field or an induced magnetic field by the solar wind. The answer has recently been given by the Mars Global Surveyor which found that the surface magnetic field of a global intrinsic magnetic field is likely to be smaller than about $5 \mathrm{nT}$ (Acuña et al., 1998). Thus, the magnetic field of core origin is unlikely to be a dominant magnetic field in the ionosphere, and the ionosphere of Mars can be considered to be filled with the magnetic field induced by the solar wind. Since the magnitude of the intrinsic magnetic field at Mars is found to be small, the nature of the solar wind-Mars interaction should be analogous to that of the solar wind-Venus interaction. The question now is whether or not theoretical models based on the idea of the solar wind-Venus interaction are able to reproduce the plasma environment of Mars.

Vertical profiles of the calculated electron density are shown in Fig. 10 along with the electron densities obtained by the Viking 1 and 2 landers. Case 1 and Case 2 are the cases for $V_{\mathrm{sw}}=450 \mathrm{~km} / \mathrm{s}$ and $300 \mathrm{~km} / \mathrm{s}$, respectively. V1 and V2 denote the electron density profiles of the Viking 1 and 2 landers, respectively. As is shown in Figs. 3, 5, and 8, both for Case 1 and Case 2 an ionopause is seen. The ionopause is created by the combination of downward motion and horizontal transport process, as was discussed for the Venus ionosphere (Cravens and Shinagawa, 1991; Shinagawa, 1996; Tanaka and Murawski, 1997; Tanaka, 1998). However, the radio occultation measurements made by the Mariner and Viking

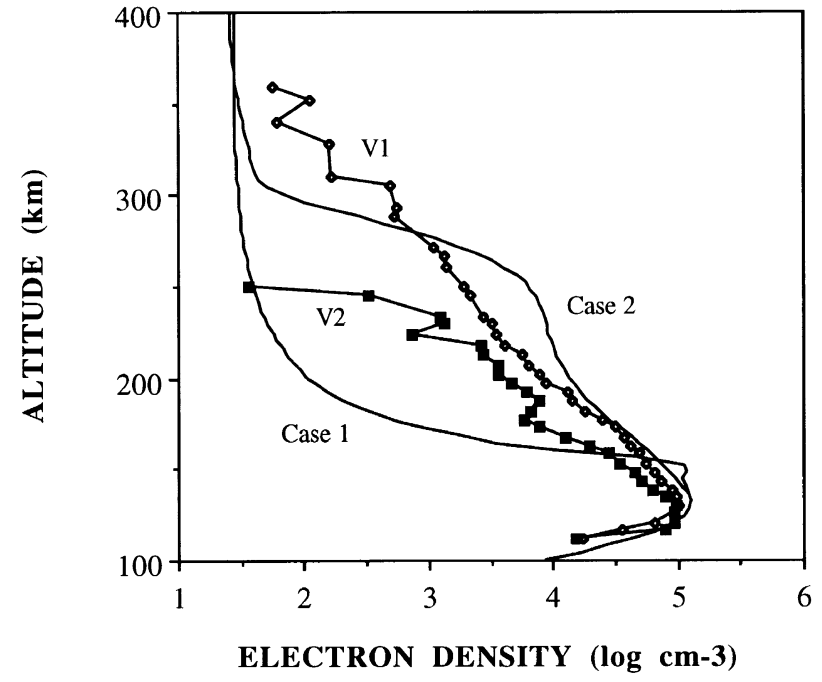

Fig. 10. Altitude profiles of the calculated electron densities at solar zenith angle $(\mathrm{SZA})=45^{\circ}$ for Case 1 and Case $2 . \mathrm{V} 1$ and V2 denote the observed electron density profiles obtained by the Viking 1 and 2 landers, respectively.

orbiters indicate that most of the observed electron density profiles have fairly constant and relatively large plasma scale height at all altitudes, exhibiting no clear ionopause structure (Zhang et al., 1990; Kliore, 1992).

In the Venus ionosphere for $P_{\mathrm{sw}}>P_{\mathrm{i}}$, a strong convection is believed to be driven in the ionosphere, giving a very small plasma scale height above the photochemical region. On the other hand, most of the electron density profiles observed obtained by previous measurements appear to be fairly stable and have fairly large and constant scale height on the dayside (Kliore, 1992). Krymskii et al. (1995) pointed out that the scale height of the Martian ionosphere should be significantly smaller than the observed one, if the magnitude of plasma convection in the Martian ionosphere is as large as that in the Venus ionosphere. A recent 3D study of Tanaka (1998) also tends to produce a smaller scale height than the observed one under the condition of $P_{\mathrm{sw}}>P_{\mathrm{i}}$, although the 3D model treats the lower part of the ionosphere rather crudely. The result in our study is consistent with the 3D study.

Recently the Mars Global Surveyor has discovered fairly strong ( $B \leq 400 \mathrm{nT}$ ) but highly localized magnetic fields which are probably of crustal origin at various places (Acuña et al., 1998). It is important to study how such localized magnetic fields affect the ionosphere and the solar wind-Mars interaction. The rotation of Mars might also play an important role in dynamics of the ionosphere. It is also expected that at solar maximum the ionospheric thermal pressure exceeds the average solar wind dynamic pressure most of the time. Under such conditions, the solar wind electric field would be effectively shielded by the ionosphere of Mars, and the crustal magnetic field might become a dominant magnetic field below the ionosphere. In addition, the upper atmosphere may also be influenced by gravity waves and tidal waves propagating from the lower atmosphere (Bougher and Shinagawa, 1998), leading to modification of the ionosphere. Those effects need to be considered in future modeling study. 


\section{Summary}

Structure of the ionosphere associated with the solar wind interaction with Mars was investigated using the 2D MHD model which includes the solar wind. Although the 2D model has various limitations in quantitatively reproducing physical processes around Mars, the modeled profiles of the electron density and the magnetic field give some idea of the ionospheric processes.

In the $2 \mathrm{D}$ model, a clear and relatively sharp ionopause is always formed in the dayside ionosphere for realistic solar wind parameters. This result is consistent with recent 3D study (Tanaka, 1998). Like the ionosphere of Venus, the ionopause altitude changes with SZA as well as the solar wind dynamic pressure. This behavior, however, is not consistent with most of the observations made by the Mariner and Viking spacecraft, which rarely observed clear ionopauselike structure. This fact suggests that processes not present in the Venus ionosphere, such as crustal magnetic fields and the rotation of the planet, may have significant effects on the structure and the dynamics of the ionosphere of Mars.

In spite of very small intrinsic magnetic field, the Martian plasma environment appears to be somewhat different from Venus. In addition to the solar wind-induced magnetic field and magnetic fields of crustal origin and/or effects of the rotation of Mars might play a significant role in determining the structure and dynamics of the ionosphere of Mars. Observations of the upper atmosphere by NOZOMI, which was launched in the summer of 1998, along with further modeling studies will give a clue to understand the nature of the solar wind interaction with Mars.

Acknowledgments. This work was done using computers of the Nagoya University Computing Center.

\section{References}

Acuña, M. H., J. E. P. Connerney, P. Wasilewski, R. P. Lin, K. A. Anderson, C. W. Carlson, J. McFadden, D. W. Curtis, D. Mitchell, H. Reme, C. Mazelle, J. A. Sauvaud, C. d'Uston, A. Cros, J. L. Medale, S. J. Bauer, P. Cloutier, M. Mayhew, D. Winterhalter, and N. F. Ness, Magnetic field and plasma observations at Mars: Initial results of the Mars Global Surveyor mission, Science, 279, 1676-1680, 1998.

Bougher, S. W. and H. Shinagawa, The Mars thermosphere-ionosphere: Predictions for the arrival of Planet-B, Earth Planets Space, 50, 247257, 1998.

Cable, S. and R. S. Steinolfson, Three dimensional MHD simulations of the interaction between Venus and the solar wind, J. Geophys. Res., 100, 21645-21658, 1995.

Cravens, T. E. and H. Shinagawa, The ionopause current layer at Venus, $J$.
Geophys. Res., 96, 11119-11131, 1991.

Dolginov, Sh. and L. N. Zhuzgov, The magnetic field and the magnetosphere of the planet Mars, Planet. Space Sci., 39, 1493-1510, 1991.

Elphic, R. C., C. T. Russell, J. G. Luhmann, F. L. Scarf, and L. H. Brace, The Venus ionopause current sheet: Thickness length scale and controlling factors, J. Geophys. Res., 86, 11430-11438, 1981.

Hanson, W. B. and G. P. Mantas, Viking electron temperature measurements: Evidence for a magnetic field in the Martian ionosphere, J. Geophys. Res. 93, 7538-7544, 1988.

Hanson, W. B., S. Sanatani, and D. R. Zuccaro, The Martian ionosphere as observed by the Viking retarding potential analyzers, J. Geophys. Res., 82, 4351-4363, 1977 .

Kliore, A. J., Radio occultation observations of the ionospheres of Mars and Venus, in Venus and Mars: Atmospheres, Ionospheres, and Solar Wind Interactions, edited by J. G. Luhmann, M. Tatrallyay, and R. O. Pepin, Geophysical Monograph Series, 66, pp. 265-276, American Geophysical Union, Washington, D.C., 1992.

Krymskii, A. M., T. Breus, and E. Nielsen, On possible observational evidence in electron density profiles of a magnetic field in the Martian ionosphere, J. Geophys. Res., 100, 3721-3730, 1995.

McGary, J. E. and D. H. Pontius, Jr., MHD simulations of boundary layer formation along the dayside Venus ionopause due to mass loading, $J$ Geophys. Res., 99, 2289-2300, 1994.

Murawski, K. and R. S. Steinolfson, Numerical simulations of mass loading in the solar wind interaction with Venus, J. Geophys. Res., 101, $2547-$ 2560, 1996a.

Murawski, K. and R. S. Steinolfson, Numerical modeling of the solar wind interaction with Venus, Planet. Space Sci., 44, 243-252, 1996b.

Schwingenschuh, K., W. Riedler, H. Lichtennegger, Ye. Yeroshenko, K. Sauer, J. G. Luhmann, M. Ong, and C. T. Russell, Martian bow shock: Phobos observations, Geophys. Res. Lett., 17, 889-892, 1990.

Shinagawa, H., A two-dimensional MHD model of the solar wind interaction with the Venus ionosphere, COSPAR Colloq. Ser., 4, 199-202, 1993.

Shinagawa, H., A two-dimensional model of the Venus ionosphere: 2. Magnetized ionosphere, J. Geophys. Res., 101, 26921-26930, 1996.

Shinagawa, H. and T. E. Cravens, A one-dimensional multi-species magnetohydrodynamic model of the dayside ionosphere of Mars, J. Geophys. Res., 94, 6506-6516, 1989.

Shinagawa, H. and T. E. Cravens, The ionospheric effects of a weak intrinsic magnetic field at Mars, J. Geophys. Res., 97, 1027-1035, 1992.

Slavin, J. A. and R. E. Holzer, The solar wind interaction with Mars revisited J. Geophys. Res., 87, 10285-10296, 1982.

Tanaka, T., Effects of decreasing ionospheric pressure on the solar wind interaction with non-magnetized planets, Earth Planets Space, 50, 259268, 1998.

Tanaka, T. and K. Murawski, Three-dimensional MHD simulations of the solar wind interaction with the ionosphere of Venus: Results of twocomponent reacting plasma simulation, J. Geophys. Res., 102, 19805$19821,1997$.

Zhang, M. H. G., J. G. Luhmann, A. J. Kliore, and J. Kim, A post-Pioneer Venus reassessment of the Martian dayside ionosphere as observed by radio occultation methods, J. Geophys. Res., 95, 14829-14839, 1990.

H. Shinagawa (e-mail: sinagawa@stelab.nagoya-u.ac.jp) and S. W. Bougher (e-mail: sbougher@1pl.arizona.edu) 